\title{
A new technique for comparing solar dynamo models and observations
}

\author{
P. D. Mininni ${ }^{1, \star}$ and D. O. Gómez ${ }^{1,2}$ \\ ${ }^{1}$ Departamento de Física, Facultad de Ciencias Exactas y Naturales, Universidad de Buenos Aires, Ciudad Universitaria, \\ 1428 Buenos Aires, Argentina \\ e-mail: mininni@df.uba.ar \\ 2 Instituto de Astronomía y Física del Espacio, CONICET, Ciudad Universitaria, 1428 Buenos Aires, Argentina \\ e-mail: dgomez@df.uba.ar
}

Received 11 March 2004 / Accepted 5 July 2004

\begin{abstract}
We present a new technique suitable for a detailed comparison between solar dynamo models and observations. The method is based on the technique of dynamo spectroscopy proposed by Hoyng \& Schutgens (1995) and bi-orthogonal decomposition of solar data. This decomposition provides a representation of the mean and fluctuating components of the flows, yielding relevant information for the comparison. To illustrate the method, we use a simple kinematic dynamo model of the solar cycle. Irregularities are introduced in the evolution of the magnetic fields modeling the turbulent behavior of the solar convective region with a random perturbation on the external source for the poloidal field. After fine tuning the parameters of the model we obtain solar like solutions displaying a magnetic cycle of 22 years, with fluctuations in its period and amplitude. In addition, the model generates Maunder-like events with a time span of 60-100 years.
\end{abstract}

Key words. Sun: magnetic fields - Sun: sunspots - magnetohydrodynamics (MHD)

\section{Introduction}

Since the pioneering papers by Parker (1955) (proposing the first theoretical mechanism for sunspot formation), Babcock (1961) (a qualitative model of the solar cycle) and Leighton (1969) (the first numerical simulation based on Babcock's scenario), our understanding of the solar dynamo has increased considerably (Dikpati \& Charbonneau 1999; Nandy \& Choudhuri 2002). Mean field theory (Steenbeck et al. 1966) provided a mathematical formalism for the dynamo problem, although many of its assumptions are still being debated. On the other hand, in the last years the number of observations available of the solar magnetic and velocity fields grew steadily. These observations led to the construction of improved models for the solar cycle, which involve many different mechanisms such as current generated by turbulent motions (Charbonneau \& MacGregor 1996), Babcock-Leighton (Durney 1995; Dikpati \& Charbonneau 1999, and references therein), or buoyant instabilities of magnetic flux tubes within the base of the convective region (Ferriz-Mas et al. 1994; Caligari et al. 1995).

Detailed models now describe magnetic field reversals every 11 years, migration of activity toward the equator as the cycle evolves, and a maximum of activity limited to a belt

* Present address: Advanced Study Program, National Center for Atmospheric Research, PO Box 3000, Boulder, Colorado 80307, USA. in latitude, among other features. However, many features are extremely difficult to model. A good example of these difficulties is the fact that sunspots only appear in a narrow belt between $\pm 30^{\circ}$ (Nandy \& Choudhuri 2002 for a detailed discussion).

Even more difficult to explain and model are the observed irregularities. From cycle to cycle, the sunspot number may vary by as much as $100 \%$, while the period displays fluctuations within the range from 9 to 13 years. Superimposed on these fluctuations are secular changes on timescales of centuries, like the Maunder minimum which took place during the second half of the seventeenth century (Ribes \& Nesme-Ribes 1993; Beer et al. 1998). To explain the nature of these irregularities, two different mechanisms have been invoked in the literature: chaos or stochasticity. The complexity of chaotic dynamo systems was studied more extensively in truncated models (Zeldovich \& Ruzmaikin 1990; Knobloch \& Landsberg 1995; Knobloch et al. 1998, and references therein) although it has been shown that the introduction of additional degrees of freedom in such truncated systems often destroys the properties of the chaotic attractor (Weiss et al. 1984; Cattaneo et al. 1996; see however Tobias 1997). Also, there are no firm indicators of the existence of chaos in the sunspot number timeseries (Carbonell et al. 1994).

On the other hand, the first numerical model of the solar dynamo was also the first one to include stochastic effects as the origin of the observed irregularities (Leighton 1969). 
Choudhuri (1992) was the first to propose that the kinetic helicity (proportional to the mean field $\alpha$-effect) must fluctuate around its mean value, with a spatial and time correlation given by the properties of giant cells in the solar convective region. In 1998 these structures were finally observed (Beck et al. 1998).

The comparison between observations and models (either chaotic or with stochastic effects) is usually done in a rather qualitative way. However, the sunspot data contain a large amount of information concerning the shape and dynamics of the spatial distribution of magnetic activity, which is not considered for the validation of the models. This problem turns out to be quite relevant, since many of the observed features of the solar cycle can indeed be reproduced either with chaotic or stochastic paradigms. The critical question is therefore: how can we validate/refute solar dynamo models displaying complex dynamics?

In previous studies we presented a set of tools to analyze the complexity in the sunspot data (Mininni et al. 2000, 2002a,b). In particular, Mininni et al. (2002b) presented a study of the temporal and spatial complexity in the sunspot data distribution using bi-orthogonal decomposition (BOD). The analysis yields evidence for a strong fluctuating component in the solar cycle and no evidence of low dimensional chaos, at least on the time scales that can be studied with these data. However, the BOD (Aubry et al. 1992; Holmes et al. 1996) can be used not only to search for complexity in a dataset, but also to characterize its geometrical properties and make comparisons with theoretical models. The first idea in this direction was proposed by Hoyng \& Schutgens (1995) in the context of a different decomposition. They introduced the name dynamo spectroscopy for the comparison between the spectral properties of solutions of the dynamo equations and decompositions into spherical harmonics of the solar magnetic fields (Stenflo \& Voguel 1986) or sunspot distribution (Gokhale 1992).

In this work we compare results from a theoretical model with observations using the BOD. To this end, we use a simple kinematic dynamo model of the solar cycle in spherical coordinates. The model is based on the one already presented in Mininni \& Gómez (2002), with some improvements in the modeling of the solar velocity profiles. In this model nonlinear effects are responsible for the saturation of magnetic fields, and a random force is introduced to model some of the observed irregularities. The model is 1D in latitude and time. To the best of our knowledge, Wang et al. (1991) were the first to present a radially averaged $1 \mathrm{D}$ model with meridional flow. While 2D models are now available (Dikpati \& Charbonneau 1999; Nandy \& Choudhuri 2002), to study irregular dynamics long exploratory runs in both hemispheres with high latitudinal resolutions must be carried out. As a result, we made a special effort to obtain a good $1 \mathrm{D}$ reduction of the dynamo equations, keeping as much information as possible from the observed solar velocity profiles, and displaying solar-like solutions. We want to point out that we are not proposing this simplified model as a physical description of the solar dynamo, but rather as a paradigmatic example in which the power of spectral comparison can be easily appreciated. In this context, a study of the effect of stochastic force in 2D models in one hemisphere with moderately long runs was carried out recently by Charbonneau \& Dikpati (2000).

The BOD is applied to the synthetic series generated by the model and to observations. A good agreement between the model and observations is found. Moreover, several features (such as a 7-year peak in the time power spectrum of the observational data) can be identified in the synthetic series, and related to physical effects such as the nonlinear saturation due to Lorentz forces. We believe that the methodology and its results must be extended to other models as well (including 2D models), to validate or refute different physical mechanisms and to build a realistic model for the solar cycle.

In Sect. 2 we introduce the BOD as a tool to study and compare spatio-temporal series. Section 3 describes our simple model and the source of irregularities in the solar cycle. Comparison between the BOD of our model and observations is not made until Sect. 4 for the sake of clarity. In addition to modeling the fluctuations observed in the period and amplitude of the cycles, our stochastically forced model spontaneously generates long periods of reduced activity. While the main aim of this work is to introduce the method used for a comparison with observations, in Sect. 5 we present a short discussion of these special solutions which resemble Maunder minimum events. Finally, in Sect. 6 we present the conclusions of our work.

\section{Bi-orthogonal decomposition}

To compare the solutions of dynamo models with the observed sunspot series, we will study the statistical properties of the magnetic fields. In previous studies this comparison was made considering only partial information provided by the observational dataset (Hoyng 1993; Dikpati \& Charbonneau 1999; Charbonneau \& Dikpati 2000). Several observational laws exist which summarize statistical properties observed in the solar cycle, such as Joy's law, Hale's law, and the correlation between phase and amplitude in the sunspot series (Hoyng 1993; Charbonneau \& Dikpati 2000). Beside checking the validity of these laws, other features such as the mean period and amplitude of the cycles, and the latitudinal belt where maximum activity takes place, are sometimes also compared (Dikpati \& Charbonneau 1999; Mininni \& Gómez 2002).

In a recent work we presented a detailed analysis of the complexity present in the sunspot number database using biorthogonal decomposition (Mininni et al. 2002b). Since the BOD is essentially a statistical decomposition, all the information summarized in the observational laws mentioned above is comprised in its results. Basically, the BOD consists of decomposing a spatio-temporal signal into an orthogonal set of temporal and spatial modes which are coupled to one another. This decomposition is performed in terms of empirical modes which are eigenvectors of a linear operator constructed using the empirical data. This projection has the property of being optimal: the empirical modes are ordered by their energy instead of their wave number (Aubry et al. 1992; Holmes et al. 1996).

Details of the decomposition can be found in Mininni et al. (2002b), and a comprehensive description can be found in 
Holmes et al. (1996). Here we outline the method used to obtain the time and spatial modes. Given a spatio-temporal (in general complex valued) signal $u(x, t)$, where $x \in X$ and $t \in T$ are respectively the space and time variables, we can define a linear operator $U: H(X) \rightarrow H(T)$ and its adjoint $U^{*}: H(T) \rightarrow H(X)$ such that

$$
\begin{aligned}
& \forall \phi \in H(X) \quad(U \phi)(t)=\int_{X} u(x, t) \phi(x) \mathrm{d} x, \\
& \forall \psi \in H(T) \quad\left(U^{*} \psi\right)(x)=\int_{T} u^{*}(x, t) \psi(t) \mathrm{d} t .
\end{aligned}
$$

$H$ is a linear Hilbert space with the standard inner product, and the asterisk denotes transpose complex conjugate operation. Assuming that $U$ is a compact operator, we can write a convergent series which represents the BOD of the signal $u(x, t)$,

$u(x, t)=\sum_{k=1} \alpha_{k} \phi_{k}^{*}(x) \psi_{k}(t)$.

From Eqs. (1) and (2), the bi-orthogonal modes $\phi(x)$ and $\psi(t)$ are solutions of the eigenvalue problem given by

$$
\begin{aligned}
& \left(U^{*} U \phi_{k}\right)(x)=\alpha_{k}^{2} \phi_{k}(x) \\
& \left(U U^{*} \psi_{k}\right)(t)=\alpha_{k}^{2} \psi_{k}(t) .
\end{aligned}
$$

Here the functions $\phi_{k}(x)$ and $\psi_{k}(t)$ are called respectively the eigenfunctions and the time traces of the decomposition, and the squared eigenvalue $\alpha_{k}^{2}$ is called the energy of the mode $k$.

In the case of a finite and discrete dataset, let us assume that the matrix $U_{i j}\left(i=1,2,3, \ldots, N_{X} ; j=1,2,3, \ldots, N_{T}\right)$ stores the intensity of the toroidal magnetic field for different latitudes and times ( $i$ labels the $N_{X}$ latitudinal bins and $j$ labels the $N_{T}$ time steps available in the dataset). In general, $N_{T} \gg N_{X}$. Then the eigenvalues and eigenvectors of the matrix $U^{*} U$ are computed (this is a small problem involving a $N_{X} \times N_{X}$ matrix). From Eq. (4) it follows that the eigenvalues obtained are the energies $\alpha_{k}^{2}$ (here $k$ labels the ordering, from larger to smaller energies), and the eigenvectors are the empirical modes denoted in the continuum case as $\phi_{k}(x)$. Therefore, for the discrete dataset the eigenfunctions $\phi_{k}(x)$ are $N_{X}$ vectors of $N_{X}$ components each.

The time traces in Eq. (5) could be obtained in the same way by solving the eigenvalue problem for the matrix $U U^{*}$. However, this is a larger matrix with $N_{T} \times N_{T}$ elements. Instead, the discrete version of Eq. (1) can be used. Therefore the $N_{X}$ vectors with $N_{T}$ elements representing the time traces $\psi_{k}(t)$ are obtained after multiplying the row vectors of the spatial eigenfunctions with the matrix $U$, except for a normalization factor.

One of the difficulties involved in the comparison between models and observations is related to the definition of mean fields. Almost all solar dynamo models include some kind of averaging to obtain axisymmetric equations (Dikpati \& Charbonneau 1999; Ossendrijver 2000). As a result, the models predict the evolution of the mean fields as a function of space and time. On the other hand, the solar magnetic field is highly intermittent. Therefore the interpretation of the results becomes intricate, since the translation from observed fields to mean fields involves an unknown filling factor
(Ossendrijver 2000). The BOD provides the most efficient way of capturing the dominant components of infinite-dimensional processes with only a few modes (Holmes et al. 1996). When applied to turbulent flows, it provides a representation of the mean and fluctuating velocity fields, and the corresponding modes can be related to two-point velocity correlations (Pope 2000). This ability to provide a representation of the mean and fluctuating component of a flow yields relevant information for the comparison of dynamo models.

\section{A simple dynamo model}

\subsection{The dynamo equations}

We will work in spherical coordinates under the kinematic approximation, i.e. we assume the velocity field to be given by $\boldsymbol{U}=u_{r}(r, \theta) \hat{r}+u_{\theta}(r, \theta) \hat{\theta}+r \sin \theta \omega(r, \theta) \hat{\phi}$, with all the velocity profiles known and where $\omega$ describes the solar differential rotation.

Below the solar tachocline, the rotation velocity inferred from helioseismology remains approximately constant, and the inner region seems to rotate like a rigid body. The latitudinal shear observed in $\omega$ is small at the base of the tachocline, while above it $\omega$ displays a profile that remains approximately independent of the radius across the convective region until reaching the surface. At the surface, the differential rotation profile $\omega_{\mathrm{s}}$ is given by observations (Beck 1999),

$\omega_{\mathrm{s}}(\theta)=a+b \cos ^{2}(\theta)+c \cos ^{4}(\theta)$,

where $a=2.913 \times 10^{-6} \mathrm{rad} \mathrm{s}^{-1}, b=-0.405 \times 10^{-6} \mathrm{rad} \mathrm{s}^{-1}$, $c=-0.422 \times 10^{-6} \mathrm{rad} \mathrm{s}^{-1}$. The meridional velocity fields $u_{r}$ and $u_{\theta}$ are assumed to be incompressible although in a stratified medium, following the expressions in Mininni \& Gómez (2002).

Considering the observed velocity field, in the kinematic approximation we must solve the induction equation to obtain the magnetic field $\boldsymbol{B}$

$\frac{\partial \boldsymbol{B}}{\partial t}=\nabla \times(\boldsymbol{U} \times \boldsymbol{B})+\eta \nabla^{2} \boldsymbol{B}$,

where $\eta$ is the magnetic diffusivity. We assume axisymmetry and write down the magnetic field in spherical coordinates in terms of its toroidal and poloidal components, i.e. $\boldsymbol{B}=B_{\phi} \hat{\phi}+\boldsymbol{B}_{\mathrm{p}}$, where $\boldsymbol{B}_{\mathrm{p}}=\nabla \times\left(A_{\mathrm{p}} \hat{\phi}\right)$.

There is strong observational evidence that suggests that dynamo action takes place at the bottom of the convective region. Therefore we reduce the induction Eq. (7) to $1 \mathrm{D}$ equations for $A_{\mathrm{p}}$ and $B_{\phi}$ as a function of latitude (Mininni \& Gómez 2002). Helioseismic measurements suggest that the meridional velocity field varies in the radial direction more smoothly than the magnetic field and differential rotation profiles. Therefore Eq. (7) is decomposed into its poloidal and toroidal components, and we take the meridional flow components $u_{r}$ and $u_{\theta}$ out of the radial derivatives and approximate them by their radially averaged values (Fig. 1).

To properly model the strong and localized radial shear observed in the differential rotation, we expand all radial derivatives in the dynamo equations. Radial derivatives of the unknowns $A_{\mathrm{p}}$ and $B_{\phi}$ were replaced by the corresponding field 


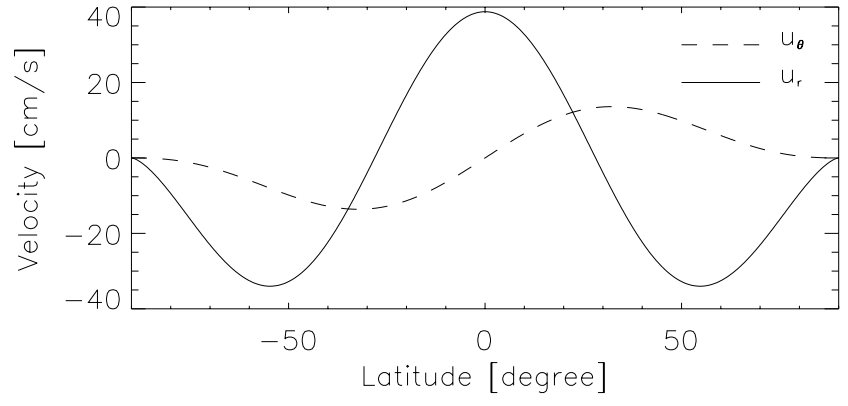

Fig. 1. Radially averaged components of the meridional flow, for a maximum meridional velocity at the surface of $20 \mathrm{~m} \mathrm{~s}^{-1}$.

component times $1 / L_{0}$ (where $L_{0}$ is the typical depth of the tachocline), while for the differential rotation we use $\partial_{r} \omega \approx$ $\left(\omega_{\mathrm{s}}-\omega_{\mathrm{c}}\right) / L_{0}=\Delta \omega / L_{0}$, where $\omega_{\mathrm{c}}$ describes the rotation of the core as a rigid body. In this way, we consider the most important property of the tachocline known from observations: that the core rotates as a rigid body, and that the differential rotation profile remains almost constant from the surface through most of the convective region.

To write down the resulting equations in dimensionless units we define dimensionless variables with the aid of a typical time $T_{0}$ (approximately 1 year), and a typical longitude $L_{0} \ll 1 R_{\odot}$. The magnetic field is expressed in velocity units. Under all these assumptions Eq. (7) finally becomes

$$
\begin{aligned}
\frac{\partial B_{\phi}}{\partial t}= & -\left(u_{r}+\epsilon \frac{\partial u_{\theta}}{\partial \theta}\right) B_{\phi}-\epsilon u_{\theta} \frac{\partial B_{\phi}}{\partial \theta} \\
& +\left(\Delta \omega \cos \theta-\sin \theta \frac{\partial \omega_{s}}{\partial \theta}\right) A_{\mathrm{p}}+\Delta \omega \sin \theta \frac{\partial A_{\mathrm{p}}}{\partial \theta} \\
& +\frac{1}{R_{\mathrm{M}}}\left(-\frac{\epsilon^{2}}{\sin ^{2} \theta}+\epsilon^{2} \cot \theta \frac{\partial}{\partial \theta}+\epsilon^{2} \frac{\partial^{2}}{\partial \theta^{2}}-1\right) B_{\phi} \\
\frac{\partial A_{\mathrm{p}}}{\partial t}= & -\left(u_{r}+\epsilon \cot \theta u_{\theta}\right) A_{\mathrm{p}}-\epsilon u_{\theta} \frac{\partial A_{\mathrm{p}}}{\partial \theta}+\alpha B_{\phi} \\
& +\frac{1}{R_{\mathrm{M}}}\left(-\frac{\epsilon^{2}}{\sin ^{2} \theta}+\epsilon^{2} \cot \theta \frac{\partial}{\partial \theta}+\epsilon^{2} \frac{\partial^{2}}{\partial \theta^{2}}-1\right) A_{\mathrm{p}}
\end{aligned}
$$

where $R_{\mathrm{M}}=L_{0}^{2} /\left(T_{0} \eta\right), \epsilon=L_{0} / R_{\odot} \approx 0.1$, and $u_{\theta}$ and $u_{r}$ are the radially averaged values of the meridional flow. A new term, controlled by the coupling constant $\alpha$ has been added to the induction equation to model the process responsible for the conversion of toroidal into poloidal magnetic field.

If the magnetic energy becomes comparable to the kinetic energy, nonlinear effects cannot be neglected. Therefore we introduce the back-reaction of the magnetic field on the $\alpha$ source term as

$$
\alpha \rightarrow \alpha(\boldsymbol{B})=\frac{\alpha}{1+B_{\phi}^{2} / B_{0}^{2}} \sin (\theta) \cos (\theta),
$$

where the latitude profile was set to fit estimates of kinetic helicity from solar observations (Kuzanyan et al. 2000), and where $B_{0}$ is a free parameter of the model related to the saturation value of the magnetic field. Magnetic flux tube simulations suggests than the saturation takes place when $B_{\phi} \approx$ $10^{4}-10^{5} \mathrm{G}$ (Choudhuri \& Gilman 1987; Fan et al. 1993; D'Silva \& Choudhuri 1993), and these values were used to estimate $B_{0}$ in the model.
The integration is done in both solar hemispheres. The boundary conditions at the poles are

$$
\begin{aligned}
& B_{\phi}(\theta=0)=B_{\phi}(\theta=\pi)=0 \\
& \left.\frac{\partial A_{\mathrm{p}}}{\partial \theta}\right|_{\theta=0}=\left.\frac{\partial A_{\mathrm{p}}}{\partial \theta}\right|_{\theta=\pi}=0 .
\end{aligned}
$$

The reduction of the dynamo equations to a $1 \mathrm{D}$ model allows us to perform long time simulations at sufficiently large latitudinal resolutions, a feature that will become crucial for obtaining the results presented in Sects. 4 and 5.

The sign of the net $\alpha$ effect has not been definitely established. The flux tube analysis of Ferriz-Mas et al. (1994) and MHD simulations of Brandenburg \& Schmitt (1998) predict a mostly positive $\alpha$ effect on the northern hemisphere. On the other hand, theoretical estimates (Parker 1987; Rüdiger \& Kichatinov 1993), other MHD simulations (Ossendrijver et al. 2001), and observational estimates (Kuzanyan et al. 2000), obtain an effective negative $\alpha$ effect in the northern hemisphere (with positive $\alpha$ at the surface in some cases). Also, Choudhuri et al. (1995) demonstrated that a meridional flow can force the equatorward migration of magnetic activity irrespective of the sign of $\alpha$. Since this discussion is beyond the goal of our model, following Ossendrijver (2000), $\alpha$ was assumed to be negative in the northern hemisphere. The set of Eqs. (8) were numerically integrated using a centered finite-difference scheme with 500 points in latitude, and a predictor-corrector method to evolve in time, with a time step of $T_{0} / 500$.

\subsection{Solar dynamo solutions}

Preliminary results of this model have been shown in Gómez \& Mininni (2003). In Fig. 2 we show contour levels of the toroidal magnetic field $B_{\phi}$ obtained as a result of integrating the equations with $\eta=3.39 \times 10^{11} \mathrm{~cm}^{2} \mathrm{~s}^{-1}, \alpha=14.02 \mathrm{~cm} \mathrm{~s}^{-1}$, and $B_{0}=10^{3}$ Gauss. Choudhuri (1992) estimates the $\alpha$ coefficient to range between $3 \mathrm{~cm} \mathrm{~s}^{-1}$ and $30 \mathrm{~cm} \mathrm{~s}^{-1}$, while Dikpati \& Charbonneau (1999) estimate $\eta \approx 3 \times 10^{11} \mathrm{~cm}^{2} \mathrm{~s}^{-1}$. The rotation of the core is $\omega_{\mathrm{c}}=2.243 \times 10^{-6} \mathrm{rad} \mathrm{s}^{-1}$. The maximum value of the toroidal field is $10^{5}$ Gauss, and the period of the cycle is close to 12 years. Also, the magnetic field at the poles shows an inversion close to the maximum of activity.

The evolution of the toroidal magnetic field is reasonably solar-like. The magnetic cycle starts at a latitude close to $60^{\circ}$. As the cycle evolves, the magnetic activity below this latitude migrates toward the equator, while magnetic field concentrations above $60^{\circ}$ migrate toward the poles, in good agreement with observations. Note that the direction of migration is partially related to the meridional flow, but also to the sign of $\alpha$ and of the derivatives of $\omega$. In particular, $\partial \omega / \partial r$ (in our model, proportional to $\omega_{s}-\omega_{\mathrm{c}}$ ) changes sign at mid latitudes while $\alpha$ has the same sign in each hemisphere. Therefore, the change in sign of the product $\alpha(\partial \omega / \partial r)$ is related to the migration direction. The region of strongest magnetic activity concentrates in a belt around $\pm 50^{\circ}$, while the maximum of magnetic activity is reached close to $30^{\circ}$.

Figure 3 shows the toroidal magnetic field with $\eta=5.75 \times$ $10^{11} \mathrm{~cm}^{2} \mathrm{~s}^{-1}$, and $\alpha=10.35 \mathrm{~cm} \mathrm{~s}^{-1}$. The period is close 


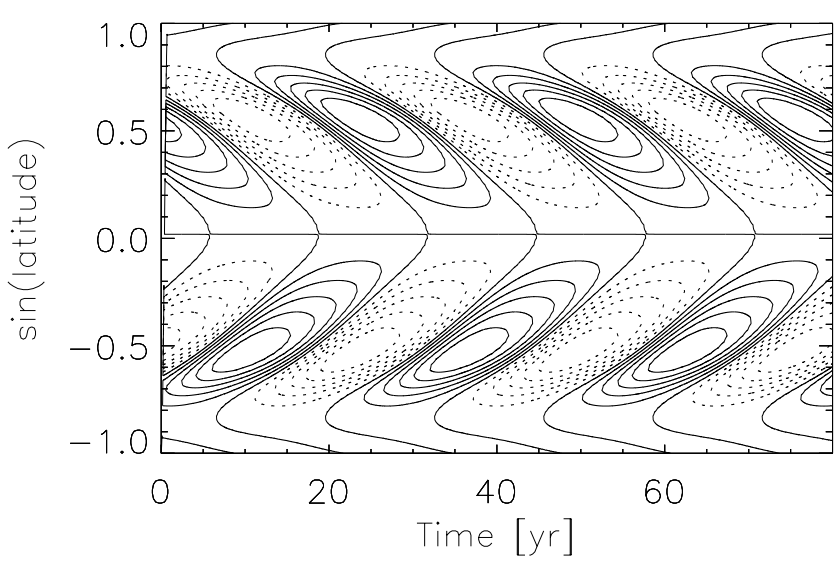

Fig. 2. Contour plot of the toroidal magnetic field in a latitudetime plane (butterfly diagram), for $\eta=3.39 \times 10^{11} \mathrm{~cm}^{2} \mathrm{~s}^{-1}, \alpha=$ $14.02 \mathrm{~cm} \mathrm{~s}^{-1}$, and $B_{0}=10^{3}$ Gauss. Full and dashed lines indicate opposite polarities. The maximum value of $B$ is $10^{5} \mathrm{G}$.

to 11 years. Here, the region of strongest magnetic activity is concentrated in a narrow belt around $\pm 40^{\circ}$, and the maximum of magnetic activity is reached at $25^{\circ}$.

The meridional flow speed has a profound influence in determining the period of the cycle, while the amplitude of $\alpha$ does not. This is in good agreement with results arising from higher dimensional codes (Dikpati \& Charbonneau 1999). However, the amplitude of $\alpha$ is related with the latitudinal spread of magnetic activity, with larger values of $\alpha$ implying a wider latitudinal belt for magnetic activity. The value of $\omega_{\mathrm{c}}$ also controls the latitude of maximum activity, since this quantity is related to the latitude where $\alpha(\partial \omega / \partial r)$ changes sign.

\subsection{Helicity fluctuations and irregularities}

As was previously mentioned, attempts to model the irregular dynamics of the solar cycle have been traditionally associated either with low dimensional chaos or stochastic effects. Studies made of the observational time series to decide between these two paradigms did not give conclusive results, since the amount of data is not large enough for statistical studies (Carbonell et al. 1994). However, several observed features of the cycle (Hoyng 1993; Charbonneau \& Dikpati 2000; Mininni et al. $2000,2002 a)$ suggest that the correct paradigm is the stochastic scenario.

What is the origin of these stochastic effects? In the outer regions of the sun, the temperature gradient is strong enough to drive convective turbulence. In mean field theory, the ergodic hypothesis made to define averages of the fields gives rise to fluctuating coefficients in the dynamo equations when the average over a statistical ensemble is replaced by an average over a finite number of convective cells. Since the number of cells is finite, fluctuations around mean values can be rather large (Choudhuri 1992; Hoyng 1993). Moreover, recent observations (Kuzanyan et al. 2000) and theoretical estimates (Choudhuri 1992; Ossendrijver \& Hoyng 1996) suggest that the amplitude of the fluctuations can be even larger than the mean field values.

In the solar dynamo, convective motions play a crucial role in the source of the poloidal field. The spatial and temporal

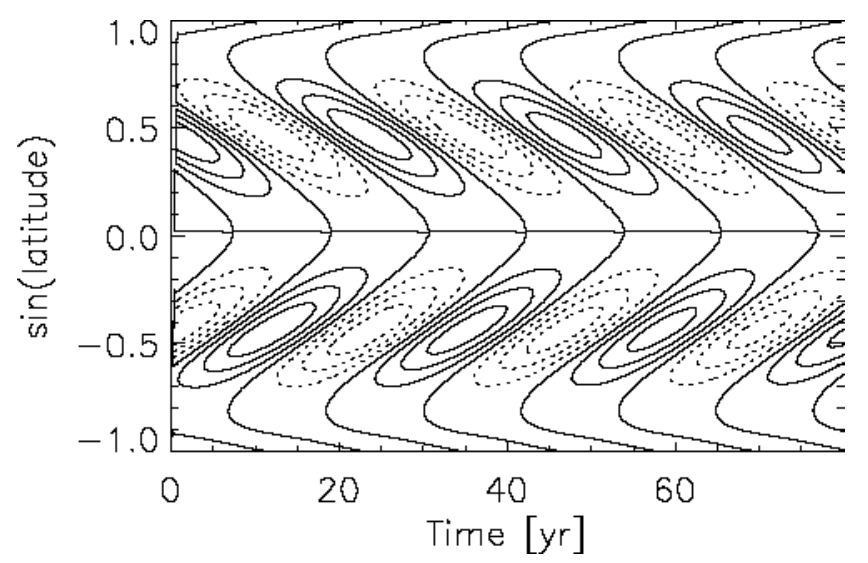

Fig. 3. Contour plot of the toroidal magnetic field in a latitudetime plane (butterfly diagram), for $\eta=5.75 \times 10^{11} \mathrm{~cm}^{2} \mathrm{~s}^{-1}, \alpha=$ $10.35 \mathrm{~cm} \mathrm{~s}^{-1}$, and $B_{0}=10^{3}$ Gauss. Full and dashed lines indicate opposite polarities. The maximum value of $B$ is $10^{4} \mathrm{G}$.

complexity of the convective cells can be described introducing a fluctuating component in the small scale velocity field, which in turn brings fluctuations into the $\alpha$ coefficient. Dynamo models with fluctuations in $\alpha$ have been studied extensively by Choudhuri (1992), and Hoyng (1993). In our model, we split $\alpha$ into a mean component $\alpha_{0}$ and a fluctuating part $\delta \alpha=r \xi$. Therefore,

$\alpha=\alpha_{0}+r \xi(\theta, t)$

where $\xi(\theta, t)$ is a Gaussian stochastic process with dispersion equal to unity. Therefore, the dimensionless parameter $r$ is the rms value of the stochastic part of $\alpha$.

What are the correlation properties of the fluctuations in $\alpha$ ? The $\alpha$ effect is related to the helical velocity field in the solar convective region, and therefore requires the reflection symmetry to be broken by an external force. In the Sun, this is done by the Coriolis force. The reciprocal of the Rosby number measures the relevance of the Coriolis force in the dynamics of the fluid

$R_{\mathrm{S}}=\frac{U}{2 L \Omega}$,

where $U$ and $L$ are characteristic velocities and lengths, and $\Omega$ is the rotation frequency $(2 \pi / \Omega \approx 27$ days). Considering $R_{\mathrm{S}} \approx 1$ we can estimate the smallest scale that will feel the effect of the Coriolis force. For convective motions with velocities of a few meters per second we obtain that the smallest relevant length scale is about $1 \times 10^{5} \mathrm{~km}$, precisely the size of the giant cells.

Recently, giant cells have been measured in the Sun and were found to be rather elongated, covering less than $10^{\circ}$ in latitude but about $40^{\circ}$ in longitude (Beck et al. 1998). Considering these results, we assume a latitudinal correlation of $8 \times 10^{4} \mathrm{~km}$ (equivalent to 9 cells in latitude), with $\delta \alpha$ spatially constant in each cell. The value of $\delta \alpha$ in each cell was randomly changed once every 60 days (the correlation time of these structures). As an example, in Fig. 4 we show the result of integrating the resulting stochastic dynamo equations, with $r=14.02 \mathrm{~cm} \mathrm{~s}^{-1}$ (i.e. $\delta \alpha / \alpha \approx 1$ ). When stochastic fluctuations are introduced, 


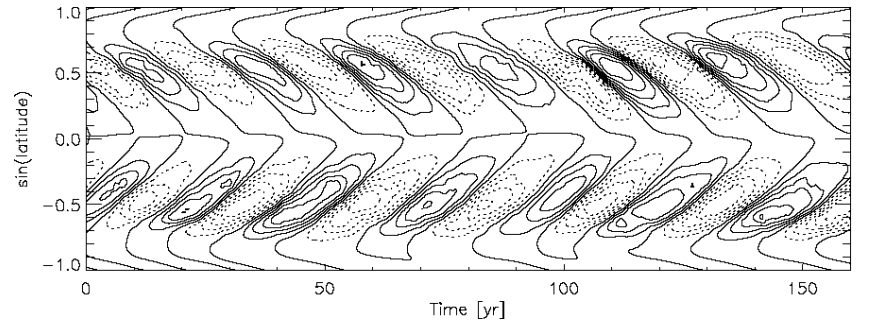

Fig. 4. Toroidal magnetic field, for a time correlation of 60 days, and a spatial correlation of $8 \times 10^{4} \mathrm{~km}$, with $r=14.02 \mathrm{~cm} \mathrm{~s}^{-1}$. Note the transequatorial activity and the asymmetry between hemispheres.

the mean period of the cycle is slightly increased, as correctly predicted in previous models and theoretical estimates (Ossendrijver \& Hoyng 1996).

While Fig. 4 displays an irregular cycle with fluctuations in amplitude and period, the cycle can be easily identified during the whole integration. In this sense, the solution shown in Fig. 4 resembles the normal irregular dynamics of the solar cycle observed in the last 300 years, with fluctuations on small scales and times but displaying a strong magnetic cycle. The cycle remains stable, even though it is strongly perturbed by noise. The solution keeps showing cyclic magnetic fields for long times (note that Fig. 4 spans for more than 150 years). It is well known that stochastically forced models can correctly describe several properties of the solar cycle, such as phase and amplitude correlation and statistical properties of the observed fluctuations (Charbonneau \& Dikpati 2000; Mininni \& Gómez 2002). On the other hand, large-scale secular events like Maunder minimum seem hard to obtain in stochastically forced models, although there are some linear (Ossendrijver et al. 1996; Ossendrijver \& Hoyng 1996) and nonlinear models (Moss et al. 1992; Ossendrijver 2000) that previously obtained results resembling these phenomena. In the next section we will focus on the normal cycle, comparing the results of numerical simulations with decompositions of the observations from 1874 . However, we want to point out that when carrying out long runs with our simple model we obtain the occurrence of grand minima, as will be shown later in Sect. 5 .

\section{Comparison between model and observations}

A BOD of the temporal and latitudinal distribution of solar magnetic fields inferred from sunspots since 1874 showed that the butterfly diagram can be interpreted as the result of two oscillations with approximately constant amplitudes and phase shift (Fig. 5c), both with periods close to 22 years (Mininni et al. 2002b). These two oscillatory modes alone comprise $84.4 \%$ of the total energy of the decomposition, and their corresponding spatial eigenfunctions are antisymmetric with respect to the equator (Fig. 5a). All the symmetric modes of the BOD in the reconstructed magnetic fields (responsible for asymmetries in the distribution of sunspots) only contain $9.6 \%$ of the energy of the system.

Auto-correlation analysis of the time traces for the first two modes shows that the series has positive correlation for time lags close to $\approx 0$ and $\approx \pm 22$ years, and negative correlation in
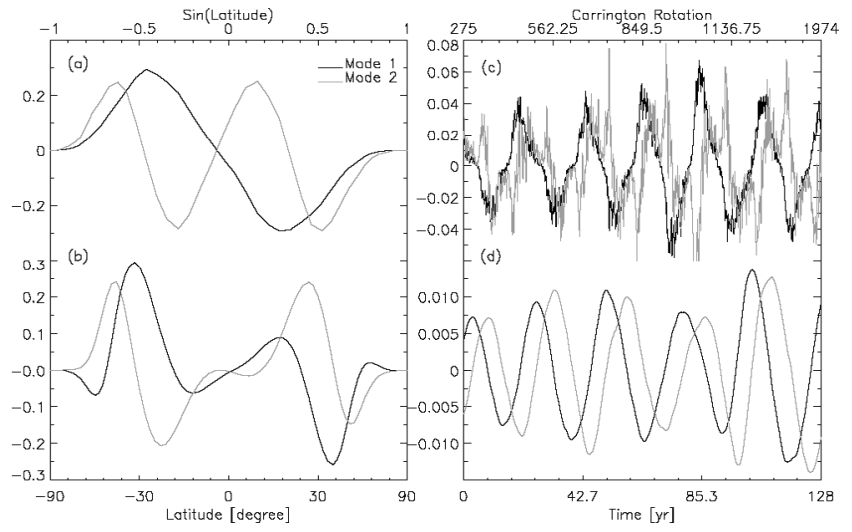

Fig. 5. First two BOD spatial eigenfunctions: a) observations, and b) model, and corresponding time traces: c) observations, and d) model.

the $\approx \pm 11$ years time lag. On the other hand, the autocorrelation of the remaining time traces falls immediately below 0.2 , which implies a strong irregular component in the rest of the modes. Also, a periodicity close to 7 years can be clearly observed in the power spectrum of the first time traces (Mininni et al. 2002b). The existence of this periodicity was considered a signature of nonlinearity in the underlying dynamical system, and has been reported in previous analyses of the sunspot time series (Bracewell 1985).

When we apply the BOD to the toroidal magnetic field given by our unperturbed model (e.g. solutions of the type shown in Figs. 2 and 3), 100\% of the energy goes into the asymmetric modes, and the first two modes comprise more than $96 \%$ of the total energy. The rest of the modes only correspond to small corrections with short length scales, while the corresponding time traces are overtones of the 22-year cycle. However, when the BOD is applied to the system perturbed by noise (Fig. 4), the first two modes have $92 \%$ of the energy, and symmetric modes appear with $7 \%$ of the total energy. This values are in good agreement with observational results. As the amplitude of the noise is increased these numbers increase smoothly. Therefore the energy distribution in the decomposition can be used to statistically adjust the amplitude of the noise. As the amplitude of the fluctuations in $\alpha$ is related to velocity fluctuations in giant cells, this result can be used to check in models the proposed link between giant cell movements and solar activity. We want to point out that in this analysis the BOD was applied to several solutions of the same type as the one shown in Fig. 4, i.e. normal cycle solutions with the same time span as the available observations (129 years). This decision is made to ensure the validity of the statistical comparison, since the data available since 1874 do not show Maunder minimum events.

Figure 5 shows a comparison of the first two modes of the BOD of observations and of the toroidal magnetic field given by our model. Several features can be identified in both decompositions. The first two time traces in both series have a period of 22 years and an almost constant phase shift of about $\pi / 2$ (Figs. 5c and d). The spatial modes also share recognizable features, such as equatorial symmetry (Figs. 5a and b). Note 


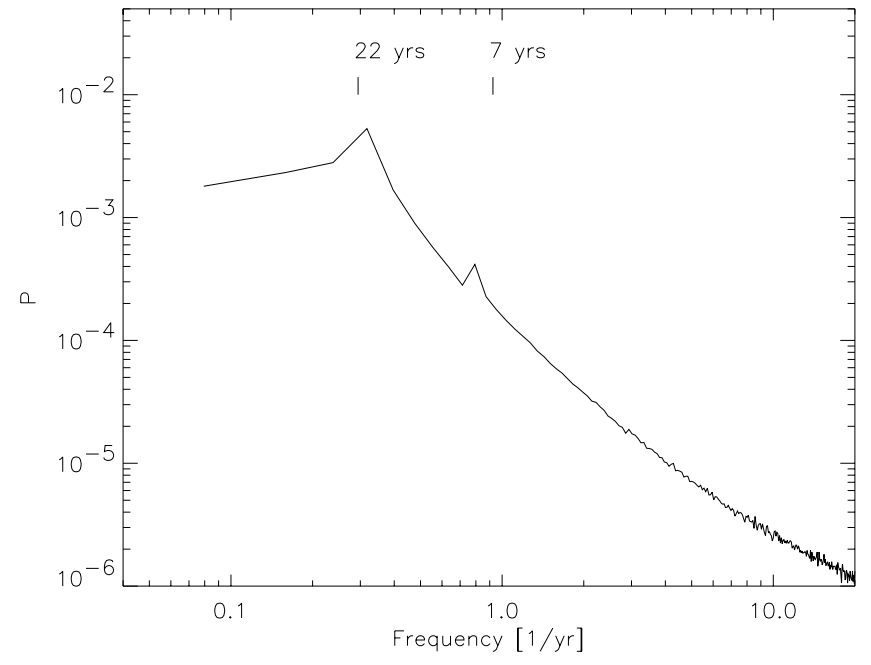

Fig. 6. Time power spectrum for the first mode of the BOD of the toroidal magnetic field generated by the model.

that in our model magnetic activity peaks at latitudes close to the observed ones. However, the distribution of magnetic fields in our model is more localized in latitude than in the solar case (the peaks in the spatial eigenfunctions are narrower). This feature can be tuned in our model by increasing the magnetic diffusivity.

The correlation coefficients for the first two model time traces show positive correlation at 0 and \pm 22 years, and negative correlation at \pm 11 years, as for the observations. The correlation for the rest of the model time traces falls quickly below 0.2 , but weak periodicities in 11 and 22 years can still be identified in these modes.

Perhaps one of the most remarkable features of the decomposition of the synthetic series is the fact that the model correctly generates the observed peak of 7 years in the time spectrum of the first two modes. Figure 6 shows the temporal power spectrum for the first time trace of the BOD of the toroidal magnetic field generated by the model. After performing several simulations with different values of the parameters in the model, we can confirm that this peak is the signature of the nonlinearity in this dynamical system. Actually, the amplitude and position of the peak in Fourier space are related to the particular nonlinearity that we used and its amplitude. For the quenching used in our model, under the approximation $\alpha(B)=\alpha_{0}\left(1+\left(B / B_{0}\right)^{2}\right)^{-1} \approx \alpha_{0}\left(1-\left(B / B_{0}\right)^{2}\right)$, the only nonlinearity in the induction equation is cubic. It is well known that a cubic nonlinearity generates a peak at $3 \omega$ due to beating (Mininni et al. 2002b), where $\omega=2 \pi / 22$ year $^{-1}$ is the fundamental frequency of the system. Therefore, the spectral properties of the BOD of the observations can be used through comparison with other models to check the correct expression for the mechanism of nonlinear saturation of the dynamo.

\section{Maunder minimum}

The solutions obtained with our model (Fig. 4) show long periods of normal magnetic activity with intense and well defined cycles. Fluctuations can be observed both in the amplitude and

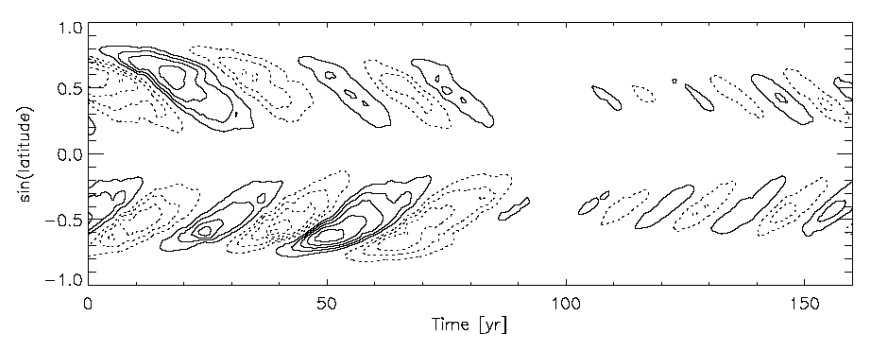

Fig. 7. Toroidal magnetic field showing occurrence of a grand minimum.

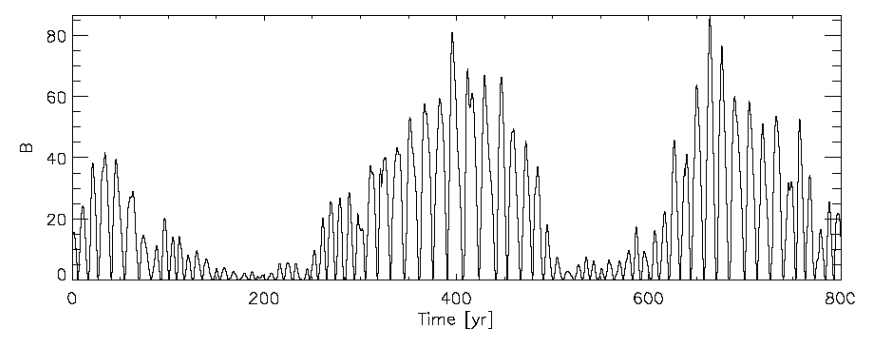

Fig. 8. Magnetic energy at mid latitudes.

in the period of the cycle, as well as slight asymmetries between hemispheres. These periods of normal activity last for long times, ranging from 100 to 700 years.

However, from time to time the modeled small scale giant cell fluctuations excite strong asymmetries between hemispheres. This can be observed in Fig. 7. When these events take place, the amplitude of the cycle decreases substantially, the period decreases, the latitudinal span of magnetic activity is also reduced, and activity enters long periods reminiscent of solar grand minima. Usually in these periods of reduced activity there is a strong asymmetry between the hemispheres, as was observed during the solar Maunder minimum by Ribes \& Nesme-Ribes (1993). As an example, in Fig. 7 the southern hemisphere is more active than the northern hemisphere for three cycles during the minimum. Note that many features observed in this simulation (such as the equatorial asymmetry, the reduction of the period, and of the latitudinal span of magnetic activity) were also observed during the Maunder minimum (Ribes \& Nesme-Ribes 1993).

These events of reduced activity typically last for 60100 years, and can be statistically observed every 400 years. The events are not periodic, and the mean time between events can be reduced by increasing the amplitude of the fluctuations.

Figure 8 shows the magnetic energy at mid latitudes contained in the toroidal magnetic field (supposed to be in phase with the sunspot number), properly normalized to be reminiscent of the observed sunspot number. Two minima events can be observed in the time series, separated by a long period of normal behavior. From abundances of ${ }^{14} \mathrm{C}$ and ${ }^{10} \mathrm{Be}$ we know that the solar Maunder minimum was probably preceded by similar episodes, the previous one taking place about 200 years earlier (Beer et al. 1998).

During the normal activity stage the energy in antisymmetric modes (in sunspots distribution) is less than $10 \%$, as mentioned in the previous section. During grand minima, on the other hand, the energy is almost equally distributed between 
symmetric and antisymmetric modes according to our simulations. This change in the symmetry of magnetic fields seems to give rise to the occurrence of Maunder minima. This origin was already suggested in the literature (Sokoloff \& Nesme-Ribes 1994; Knobloch \& Landsberg 1995; Knobloch et al. 1998) although the catalyst of this change in the symmetry is not clear. Our model shows that giant cell statistical properties can generate this change, giving as a result solutions which display an alternation of periods of normal activity and minima events.

The initial condition we use for the numerical integration is symmetric around the equator. The introduction of noise turns on overtones much weaker than the fundamental solution, but with mixed parity. The nonlinearity in the dynamo equation naturally couples this high frequency and these mixed-parity modes with the fundamental mode, thus generating this alternation between the parity of the solutions. The mixed parity solutions seem to be unstable, since minima last for a finite time and take place before the system again reaches the almost symmetric state. Note that this origin of reduced activity is different from models with thresholds for the emergency of magnetic fields, which integrate over only one hemisphere (Ossendrijver 2000).

\section{Discussion}

We present a technique suitable for a detailed comparison between solar dynamo models and observations. The method, which can be applied to 2D models as well, consists of performing bi-orthogonal decompositions of the solar observations and the results obtained from the model, to carry out a spectral comparison between both data sets.

To this end we use a simple kinematic dynamo model of the solar cycle. The dynamo equations in spherical coordinates were reduced to a $1 \mathrm{D}$ system for the toroidal and poloidal components of the magnetic field, with a special effort to preserve as much information as possible from the observed solar velocity profiles. Irregularities were introduced modeling the turbulent behavior of the solar convective region with a random perturbation in the source of the poloidal field. The statistical properties of the perturbation are chosen to match correlations observed in the giant cells.

As a result, we obtain solar-like solutions, displaying a 22 years magnetic cycle, magnetic activity concentrated in a belt, migration of the activity to the equator, and inversion of the polar magnetic field during the maximum of the cycle. The cycles are irregular and display fluctuations in period and amplitude. When performing long simulations, we find that these periods of normal activity alternate with periods of reduced activity reminiscent of the solar Maunder minimum.

The 1D model introduced in Sect. 3 is very simple, and it is only intended as an example to illustrate the use of BOD techniques to compare data and theory. However, we want to point out one issue that 2D models must address before the comparison can be performed. The BOD allows a quantitative study of symmetries, while 2D models tend to generate unrealistically symmetric magnetic fields (Ossendrijver 2000; Dikpati \& Gilman 2001). To overcome this, most 2D models impose antisymmetry and confine the integration to one hemisphere.
It has been proposed that changing the spatial location of the $\alpha$-effect could overcome this problem (Dikpati \& Gilman 2001). Also recent 3D simulations display the correct symmetry (Zhang et al. 2003). Integrations in both hemispheres must be performed for the comparison to be meaningful. We believe that the statistical and spectral comparison will allow the validation or refutation of different physical scenarios of the solar dynamo, and will make it possible to achieve the construction of a realistic dynamo model with prediction capability.

Acknowledgements. This research has been funded by grant X209 from the University of Buenos Aires. P.D.M. is a fellow of CONICET, and DOG is a member of the Carrera del Investigador Cientifico of CONICET.

\section{References}

Aubry, N., Guyonnet, R., \& Lima, R. 1992, J. Nonlinear Sci., 2, 183 Babcock, H. W. 1961, ApJ, 133, 572

Beck, J. G. 1999, Sol. Phys., 191, 47

Beck, J. G., Duvall Jr, T. L., \& Scherrer, P. H. 1998, Nature, 394, 653

Beer, J., Tobias, S., \& Weiss, N. 1998, Sol. Phys., 181, 237

Bracewell, R. N. 1985, Aust. J. Phys., 38, 1009

Brandenburg, A., \& Schmitt, D. 1998, A\&A, 338, L55

Caligari, P., Moreno-Insertis, F., \& Schüssler, M. 1995, ApJ, 441, 886

Carbonell, M., Oliver, R., \& Ballester, J. L. 1994, A\&A, 290, 983

Cattaneo, F., Hughes, D. W., \& Kim, E. 1996, Phys. Rev. Lett., 76, 2057

Charbonneau, P., \& Dikpati, M. 2000, ApJ, 543, 1027

Charbonneau, P., \& MacGregor, K. B. 1996, ApJ, 473, L59

Choudhuri, A. R. 1992, A\&A, 253, 277

Choudhuri, A. R., \& Gilman, P. A. 1987, ApJ, 316, 788

Choudhuri, A. R., Schüssler, M., \& Dikpati, M. 1995, A\&A, 303, L29

Durney, B. R. 1995, Sol. Phys., 160, 213

Dikpati, M., \& Charbonneau, P. 1999, ApJ, 518, 508

Dikpati, M., \& Gilman, P. 2001, ApJ, 559, 428

D'Silva, S., \& Choudhuri, A. R. 1993, A\&A, 272, 621

Fan, Y., Fisher, G. H., \& Deluca, E. E. 1993, ApJ, 405, 390

Ferriz-Mas, A., Schmitt, D., \& Schüssler, M. 1994, A\&A, 289, 949

Gokhale, M. H., Javaraiah, J., Kutty, K. N., \& Varghese, B. A. 1992, Sol. Phys., 138, 35

Gómez, D. O., \& Mininni, P. D. 2003, Physica A, 327, 54

Holmes, P., Lumley, J. L., \& Berkooz, G. 1996, Turbulence, coherent structures, dynamical systems, and symmetry (Cambridge: Cambridge University Press)

Hoyng, P. 1993, A\&A, 272, 321

Hoyng, P., \& Schutgens, N. A. J. 1995, A\&A, 293, 777

Knobloch, E., \& Landsberg, A. S. 1995, MNRAS, 278, 294

Knobloch, E., Tobias, S. M., \& Weiss, N. O. 1998, MNRAS, 297, 1123

Kuzanyan, K., Bao, S., \& Zhang, H. 2000, Sol. Phys., 191, 231

Leighton, R. B. 1969, ApJ, 156, 1

Mininni, P. D., \& Gómez, D. O. 2002, ApJ, 573, 454

Mininni, P. D., Gómez, D. O., \& Mindlin, G. B. 2000, Phys. Rev. Lett., 85,5476

Mininni, P. D., Gómez, D. O., \& Mindlin, G. B. 2002a, Sol. Phys., 208, 167

Mininni, P. D., Gómez, D. O., \& Mindlin, G. B. 2002b, Phys. Rev. Lett., 89, 061101

Moss, D., Brandenburg, A., Tavakol, R., \& Tuominen, I. 1992, A\&A, 265,843 
Nandy, D., \& Choudhuri, A. R. 2002, Science, 296, 1671

Ossendrijver, A. J. H., \& Hoyng, P. 1996, A\&A, 313, 31

Ossendrijver, A. J. H., Hoyng, P., \& Schmitt, D. 1996, A\&A, 313, 938

Ossendrijver, M. A. J. H. 2000, A\&A, 359, 364

Ossendrijver, M., Stix, M., \& Brandenburg, A. 2001, A\&A, 376, 713

Parker, E. N. 1955, ApJ, 121, 491

Parker, E. N. 1987, Sol. Phys., 110, 11

Pope, S. B. 2000, Turbulent flows (Cambridge: Cambridge University Press)

Ribes, J. C., \& Nesme-Ribes, E. 1993, A\&A, 276, 549

Rüdiger, G., \& Kichatinov, L. L. 1993, A\&A, 269, 581

Sokoloff, D., \& Nesme-Ribes, E. 1994, A\&A, 288, 293
Steenbeck, M., Krause, F., \& Rädler, K. H. 1966, Z. Naturforsch., 21a, 369

Stenflo, J. O., \& Vogel, M. 1986, Nature, 319, 285

Tobias, S. M. 1997, A\&A, 322, 1007

Wang, Y.-M., Sheeley, N. R., Jr., \& Nash, A. G. 1991, ApJ, 383, 431

Weiss, N. O., Cattaneo, F., \& Jones, C. A. 1984, Geophys. Astrophys. Fluid Dyn., 30, 305

Zeldovich, Ya. B., \& Ruzmaikin, A. A. 1990, Magnetic Fields in Astrophysics (New York: Gordon and Breach)

Zhang, K., Chan, K. H., Zou, J., Liao, X., \& Schubert, G. 2003, ApJ, 596,663 